\title{
Training and transfer of training in the search for camouflaged targets
}

\author{
WALTER R. BOOT \\ Florida State University, Tallahassee, Florida \\ AND \\ Mark B. Neider and Arthur F. Kramer \\ University of Illinois at Urbana-Champaign, Champaign, Illinois
}

\begin{abstract}
How do observers become proficient at finding camouflaged targets? Does gaining proficiency in locating a target in one camouflage search situation improve performance in novel camouflage situations? We examined these questions by having participants first perform a difficult camouflage search task for three sessions and, in the last session, search for novel camouflaged targets. Near-perfect transfer of training was observed, with participants finding targets in novel camouflage situations almost as quickly as in highly familiar search situations. Previous research has suggested that an "object search" strategy rather than a "background search" strategy is one reason camouflage search can be slow and inaccurate. Eye movement analyses suggest that a more background-focused strategy was not necessary for improved performance and was not responsible for transfer. Another experiment explicitly instructed participants to search background regions, rather than regions containing salient objects, and provided participants feedback regarding the location of their own fixations. Surprisingly, even in this condition, participants did not devote more oculomotor attention to background regions. Theoretical and practical implications are discussed. Supplemental figures for this article can be downloaded from app.psychonomic-journals.org/content/supplemental.
\end{abstract}

How do humans go about finding a search target when, through design (e.g., a military tank in the desert) or evolution (e.g., a lion in a savanna), it blends in with the background? Visual search has been extensively studied and a great deal has been learned about the guidance of attention during search, including how attention is controlled by bottom-up factors (e.g., Proulx, 2007; Theeuwes, 1992, 2004; Yantis \& Jonides, 1984), top-down guidance (e.g., Bacon \& Egeth, 1994; Chen \& Zelinsky, 2006; Folk, Remington, \& Johnston, 1992), and various memory mechanisms (e.g., Boot, McCarley, Kramer, \& Peterson, 2004; Brockmole \& Henderson, 2006; Chun \& Jiang, 1998; Klein \& MacInnes, 1999; Neider \& Zelinsky, 2006b). Much of what we know about search has been derived from experiments in which target and distractors are easy to segment from the background (see Wolfe, 1994, for a review). However, many real-world search situations contain items that need to be segmented from the background to some degree to allow for efficient visual search. This is especially true in the search for camouflaged targets.

As outlined by Wolfe, Oliva, Horowitz, Butcher, and Bompas (2002), the visual system faces a number of potentially important challenges when target and distractor objects are placed within the context of a visually complex background. These problems include more difficult preattentive segmentation of objects from the background and the creation of "illusory objects" by noise in the background. Additionally, if segmentation is imperfect, attentional selection may be more difficult and time consuming. Imperfect segmentation might also result in noisy object representations being passed along to higher visual stages, such as those responsible for object recognition. Through a series of cleverly designed search experiments, Wolfe and colleagues found that the main cost of background complexity was associated with delayed information accrual affecting later target recognition stages. However, in extreme cases of item-background similarity, item-by-item selection was slowed. Importantly to the present context, background complexity had a negative impact on search performance in all experiments, highlighting the difficulties associated with complex searches in which the search items are not easily distinguished from the background.

To follow up on the findings of Wolfe et al. (2002), Neider and Zelinsky (2006a) directly examined the effect of target-background similarity. Neider and Zelinsky (2006b) had participants search through displays containing many children's toys and asked them to indicate whether or not a specific toy was present. Camouflage displays were created by taking a square-shaped portion of the target image and repeating it again and again to create a background. Search items were then superimposed on this background (Figure 1). This created a search task in which the features

W. R. Boot, boot@psy.fsu.edu 


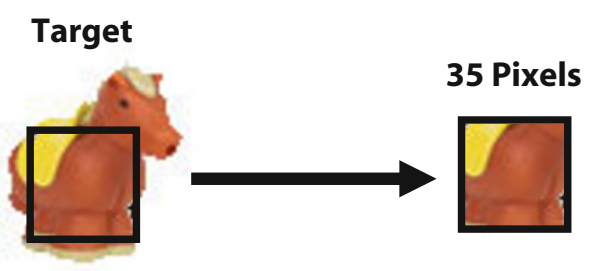

Noncamouflage Search

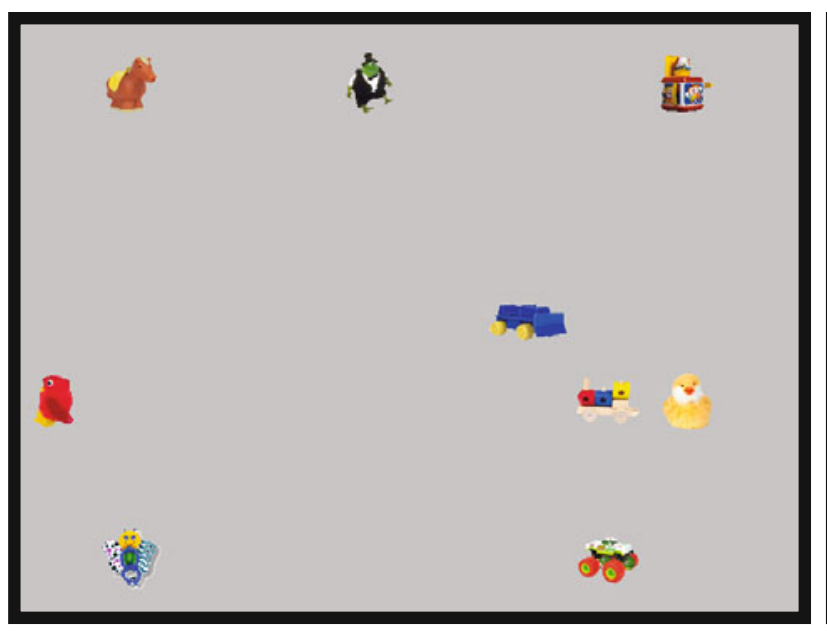

Camouflage Search

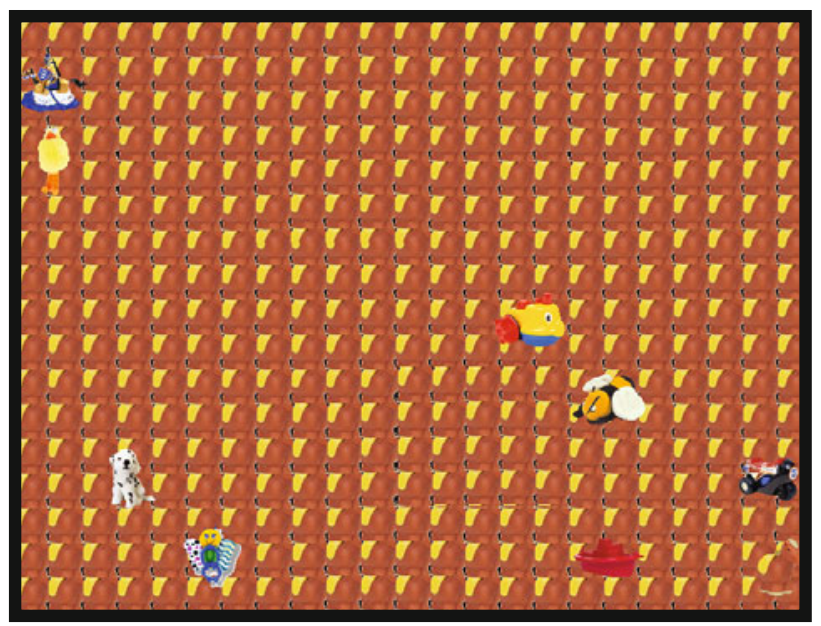

Figure 1. An example of the types of search displays participants viewed, with and without a camouflage background. In this example, the horse target is in the upper left-hand corner of the noncamouflage display and in the lower right-hand corner of the camouflage display.

of the background were highly correlated with those of the target. Target-background similarity was increased or decreased by varying the size of the square portion taken from the target image. Constructing a background using a large portion of the target toy created a background highly similar to the target; using a small portion of the target decreased the similarity between the target and the background.

Neider and Zelinsky (2006a) found that, as targetbackground similarity increased, so did response times (RTs) and error rates. As in the findings of Wolfe et al. (2002), search efficiency (i.e., search slopes) did not change with increasing target-background similarity. However, although Wolfe and colleagues (2002) ruled out a "false distractor" hypothesis as one factor contributing to search difficulty, Neider and Zelinsky (2006a) found that observers made more fixations to the background as target-background similarity increased. This result suggests that participants may have been mistaking portions of the background for potential targets. However, it is important to remember a critical difference between the search paradigms of Wolfe and colleagues (2002) and Neider and Zelinsky (2006a). Rather than the background being visually similar to target and distractor items, the background was, for the most part, similar only to the target in the experiments of Neider and Zelinsky (2006a). Thus, Neider and Zelinsky suggest that a "background search" strategy in which attention is allocated to the background might lead to efficient search. It is also important to distinguish the type of search situation studied by Neider and Zelinsky (2006a) from situations in which there are no distractor objects and observers search for a discontinuity in a textured background (Clarke, Green, Chantler, \& Emrith, 2008).

Interestingly, although participants sometimes fixated the background while searching for camouflaged targets, they were reluctant to do so. A large proportion of eye movements were directed to salient distractor items that clearly stood out from the background even though the target, by definition, was of low salience. This finding is puzzling, given that many models and theories of search propose that target features can be used to guide attention to potential target locations (e.g., Motter \& Belky, 1998; Wolfe, 1994; Zelinsky, 1996, 2008). On the basis of these models, a great many fixations would be expected on the feature-similar background. To explain this discrepancy, Neider and Zelinsky (2006a) proposed feature guidance applied largely to objects and salient discontinuities. This continuity-restricted model of feature guidance can explain both participants' tendency to fixate salient objects in the search for low-salience targets and the occasional fixation on background regions.

Given the obvious difficulty of camouflage search, and the real-world relevance of many camouflage search situations, an understanding of how to improve camouflage search performance (and the mechanisms responsible for improvement) is an important topic of research. Furthermore, one can ask whether the improvements gained with camouflage search experience transfer to novel situations; for example, does the experience a soldier gains locating 
and identifying camouflaged targets in a desert transfer to a jungle? In even simple visual perceptual tasks, transfer of training is often narrow and limited to the specific stimuli trained (e.g., Ahissar \& Hochstein, 1996; Ball \& Sekuler, 1982; Ellison \& Walsh, 1998; Fiorentini \& Berardi, 1981). Camouflage search training may demonstrate similar specificity; that is, during camouflage training, participants may learn how to detect specific targets against specific backgrounds. Alternatively, and more ideally, participants may learn something about camouflage search in general and utilize that skill, or set of skills, in the search for novel camouflage targets. For example, participants may learn to devote more attention to the feature-similar background rather than to salient distractors. However, if searchers do not naturally adopt this generalizable strategy, it may also be possible to instruct them to do so, depending on how amenable search strategy is to top-down control.

A training experiment examined improvements in camouflage search over multiple sessions and whether improvements transfer to novel camouflaged targets. This experiment utilized the same camouflage paradigm as did Neider and Zelinsky (2006a). Initially, participants were trained to search for either camouflaged or noncamouflaged targets. At the end of training, all participants were asked to search for camouflaged targets they had not seen previously. Eye movements, RTs, and accuracy were recorded. Given the aforementioned specificity of perceptual learning and visual search training, a high degree of transfer to novel targets, distractors, and backgrounds was not expected. However, to examine factors that might influence transfer of training, we varied training set variability (the number of targets participants searched for during training), predicting that the more exemplars of camouflaged targets participants encountered, the greater the transfer might be. A second experiment examined the degree to which participants can intentionally devote attentional resources to searching the target-similar background instead of the target-dissimilar but highly salient distractor items. If fixation locations during search are largely under topdown control, this should be an easy task and a large and significant difference should be observed when, unlike participants given no instruction, participants are explicitly asked to devote attention to the background regions. However, if attention allocation during search is largely salience based, little difference should be observed in the proportion of fixations on the background. Results are discussed in terms of top-down and bottom-up models of attention and search.

\section{EXPERIMENT 1}

In Experiment 1, performance and eye movements were monitored as participants gained several hours of practice searching for camouflaged or noncamouflaged targets. Some participants searched for a few targets (5), and others searched for many (20) during this training period. At transfer, all participants searched camouflage displays that contained novel targets, backgrounds, and distractors.
If camouflage-trained participants were learning general skills, they should outperform noncamouflage-trained participants at transfer. Camouflage-trained participants should also demonstrate little or no drop in performance from the last day of training to the transfer session. Furthermore, if searching for more exemplars of camouflaged targets increases the likelihood of transfer, participants who searched for 20 different camouflaged targets should outperform participants who searched for only 5 .

\section{Method}

\section{Participants}

Thirty-two students at the University of Illinois participated in Experiment 1. All participants demonstrated normal or correctedto-normal acuity and color vision. Participants were paid $\$ 32$ for their participation in four 40 - to 60 -min sessions that took place over several days.

\section{Apparatus and Stimuli}

Displays were presented on a 21-in. CRT monitor with a resolution of $800 \times 600$ pixels. An Eyelink II eyetracking system (SR Research, Inc.) sampled the position of each participant's left eye every $2 \mathrm{msec}$. An eye movement was classified as a saccade if it exceeded $0.2^{\circ}$ and either its velocity reached $30 \% \mathrm{sec}$ or its acceleration reached $9,500^{\circ} / \mathrm{sec}^{2}$. Participants viewed the monitor from a distance of approximately $62 \mathrm{~cm}$. A chinrest stabilized head position and kept viewing distance constant. A Microsoft video game controller was used to collect responses.

Stimuli were nearly identical to those used by Neider and Zelinsky (2006a). Search items were selected from the Hemera Photo Objects database. Targets and distractors were 50 pictures of toys, including dolls, stuffed animals, blocks, and toy vehicles. Each toy image was scaled to fit within an $80 \times 80$ pixel $\left(3.3^{\circ} \times 3.3^{\circ}\right)$ bounding box (Figure 1). A camouflage background for each toy was created by taking a $35 \times 35$ pixel square from the center of the toy image and tiling it across an $800 \times 600$ pixel background. In noncamouflaged displays, toys were superimposed on a gray background.

On each trial, the target was randomly selected from the list of potential training objects ( 5 objects for some participants, 20 objects for others). Distractors were randomly selected from a pool of 19 objects composed of the remaining nontarget objects within the training set (and 15 additional items for the participants training on 5 objects; exact details are described in the next section). Targets and distractors were assigned locations randomly within cells of a $10 \times 7$ imaginary grid that covered the entire display, with the exception that no object could be assigned to the center six cells in this grid. This prevented participants from fixating the target at the start of the trial.

\section{Design and Procedure}

Participants completed three sessions of training with 400 trials per session for a total of 1,200 training trials. After training, participants completed one session of 400 transfer trials. Two separate sets of 20 objects were created for training and transfer sessions; these two sets of objects will be referred to as Set A and Set B. Half of all participants were trained on objects from Set A, and at transfer searched displays contained objects from Set B. The other half were trained on objects from Set B and were tested at transfer on Set A. This was done to equate for any differences in search difficulty during training and transfer. Half of all participants were trained on all 20 objects as potential targets from a set during training. The other half were trained on a subset of 5 objects as targets from a set. ${ }^{1}$ Some participants searched for camouflaged targets during training, and others searched for noncamouflaged targets. This resulted in four groups: (1) noncamouflage-trained 5-exemplar group; (2) noncamouflage-trained 20-exemplar group; (3) camouflage- 
trained 5-exemplar group; and (4) camouflage-trained 20-exemplar group. There were 8 participants in each group.

Participants were asked to press one button on the game controller if the target was present and another if it was absent. On half of all trials, the target was present. Set size (the number of objects in the display) was equally divided between set sizes of 9 and 19 objects. Participants were asked to respond as quickly as possible while not sacrificing accuracy for speed. Feedback regarding speed and accuracy was provided every 20 trials.

Each trial began with the participant looking at a fixation dot at the center of the screen and pushing a button to initiate the trial. Immediately afterward, the search target was presented on a white background in the middle of the screen. After $1 \mathrm{sec}$, the target was replaced with the search display. The search display was removed when a response was made and a message indicated whether participants were correct or not.

\section{Results}

The first 20 trials of each session were considered practice. Due to the large amount of training data, only critical effects and interactions are discussed and analyses will focus on differences between initial training performance and performance at the end of training (Sessions 1 vs. 3). Analyses focus on training, then on transfer sessions. Figures 2 and 3 depict RT and accuracy for each condition and group for Session 1 and Session 3. Table 1 lists RT and accuracy data for each session.

\section{Training Improvements: RTs (Sessions 1 vs. 3)}

Visual inspection of RT data suggests that, although all groups responded faster with training, camouflage groups clearly benefited most (Figure 2). RTs were entered into an ANOVA with set size ( 9 vs. 19), session (1 vs. 3 ), and target presence (absent vs. present) as within-participants factors, and number of exemplars (5 vs. 20) and camouflage presence (absent vs. present) as between-participants factors. Of primary interest are the effects of camouflage and the number of target exemplars on training. A complex pattern of results was obtained in which there was a significant interaction between session, target presence, set size, exemplar number, and camouflage $[F(1,28)=$ 4.64, $p<.05]$. To interpret this interaction, we analyzed the noncamouflage and camouflage groups separately.

Noncamouflage groups. RTs were entered into an ANOVA with set size (9 vs. 19), session (1 vs. 3 ), and target presence (absent vs. present) as within-participants factors and number of target exemplars (5 vs. 20) as a between-participants factor. In addition to typical visual search effects (faster RTs for smaller set size and when the target is present), session, set size, and exemplar number interacted $[F(1,14)=6.39, p<.05]$, with a trend for session, target presence, set size, and exemplar number to interact $[F(1,14)=4.39, p=.055]$. It is clear in the right half of Figure 2 that RTs improved more for noncamouflage-trained participants who only searched for a small set of objects than for participants who searched for a large number of targets that repeated less frequently. This was especially true in the large set size target-absent condition, explaining the session, set size, and exemplar number interaction. However, the conclusion that greater improvement (in terms of RT) is the result of searching for a smaller set of objects must be tentative, given the initially slower performance of this group (see Figure 2).

Camouflage groups. RTs for camouflage-trained participants were entered into an identical ANOVA. Large improvements in RTs were observed (compared with the relatively modest improvements of noncamouflage groups). Unlike the noncamouflage group, it appears that the number of target exemplars made little difference in training camouflage search. This is evident from Figure 2 and the lack of a significant effect of exemplar number $[F(1,14)=0.29, p=.60]$, or significant interaction between exemplar number and any other factor (all $p \mathrm{~s}>$ .14). Thus, although fewer target exemplars might have improved RTs when no background was present, this did not improve performance under camouflage conditions.

\section{Training Improvements: Accuracy \\ (Sessions 1 vs. 3)}

Visual inspection of accuracy data suggests that camouflage-trained participants performed less accurately, mostly when the target was present, and especially when the target was present and the set size was large. Accuracy data were entered into an ANOVA with set size (9 vs. 19), session (1 vs. 3), and target presence (absent vs. present) as within-participants factors and number of exemplars (5 vs. 20), and camouflage presence (absent vs. present) as between-participants factors. Similar to RT data, a complex pattern of results was observed in accuracy performance in which there was a significant interaction between session, target presence, set size, exemplar number, and camouflage $[F(1,28)=4.06, p=.05]$. To interpret this interaction, we analyzed the noncamouflage and camouflage groups separately.

Noncamouflage groups. An ANOVA on accuracy data alone, when the target was present, revealed a significant effect of session $[F(1,14)=4.80, p<.05]$. Participants who searched for noncamouflaged targets tended to respond less accurately with training; this is not entirely surprising. Participants were told to try to improve speed of detection with training. Given the relative ease with which they found the target when it was not camouflaged, the only method participants could use to improve speed was to sacrifice some degree of accuracy, although they were encouraged not to do so in the instructions.

Camouflage groups. An ANOVA on accuracy data alone, when the target was present, revealed a significant session $\times$ set size $\times$ target presence interaction $[F(1,14)=$ $4.90, p<.05]$. From Figure 3, the source of this interaction is clear. With training, camouflage-trained participants improved in terms of accuracy, especially when the target was present and the set size was large. Thus, camouflage training was successful. RT and accuracy improved for camouflage-trained participants with training.

\section{Transfer Effects (Session 4)}

Of critical interest is performance at transfer between the four different training groups. RT data (Figure 4) were entered into an ANOVA with set size (9 vs. 19) and target presence (absent vs. present) as within-participants fac- 
Table 1

Data for Each Group and Each Session of Experiment 1

\begin{tabular}{|c|c|c|c|c|c|c|c|c|c|c|c|c|}
\hline & \multicolumn{6}{|c|}{ Noncamouflage } & \multicolumn{6}{|c|}{ Camouflage } \\
\hline & RT & $S E$ & $\mathrm{ACC}$ & $S E$ & BackFix & $S E$ & RT & $S E$ & $\mathrm{ACC}$ & $S E$ & BackFix & $S E$ \\
\hline \multicolumn{13}{|c|}{20 Exemplar } \\
\hline \multicolumn{13}{|c|}{ Target Present, Session 1} \\
\hline Set size 9 & 813 & 49 & .92 & .02 & .55 & .03 & 1,862 & 151 & .92 & .02 & .66 & .01 \\
\hline Set size 19 & 995 & 53 & .93 & .01 & .47 & .01 & 2,280 & 202 & .83 & .03 & .55 & .02 \\
\hline \multicolumn{13}{|c|}{ Target Absent, Session 1} \\
\hline Set size 9 & 1,100 & 102 & .98 & .00 & .54 & .02 & 4,624 & 412 & .99 & .00 & .73 & .01 \\
\hline Set size 19 & 1,682 & 132 & .98 & .00 & .46 & .02 & 5,337 & 476 & .99 & .00 & .59 & .02 \\
\hline \multicolumn{13}{|c|}{ Target Present, Session 2} \\
\hline Set size 9 & 732 & 44 & .93 & .02 & .63 & .03 & 1,414 & 110 & .91 & .03 & .68 & .02 \\
\hline Set size 19 & 931 & 65 & .91 & .02 & .53 & .02 & 1,696 & 135 & .85 & .02 & .54 & .02 \\
\hline \multicolumn{13}{|c|}{ Target Absent, Session 2} \\
\hline Set size 9 & 969 & 90 & .98 & .01 & .61 & .02 & 3,156 & 369 & .99 & .00 & .73 & .02 \\
\hline Set size 19 & 1,559 & 187 & .97 & .01 & .54 & .02 & 3,596 & 355 & .99 & .00 & .58 & .02 \\
\hline \multicolumn{13}{|c|}{ Target Present, Session 3} \\
\hline Set size 9 & 692 & 49 & .89 & .03 & .61 & .02 & 1,283 & 96 & .92 & .02 & .68 & .03 \\
\hline Set size 19 & 880 & 64 & .90 & .02 & .55 & .02 & 1,572 & 86 & .88 & .03 & .56 & .02 \\
\hline \multicolumn{13}{|c|}{ Target Absent, Session 3} \\
\hline Set size 9 & 870 & 110 & .98 & .01 & .63 & .02 & 2,929 & 303 & 1.00 & .00 & .75 & .02 \\
\hline Set size 19 & 1,435 & 181 & .96 & .01 & .55 & .02 & 3,465 & 312 & .99 & .00 & .60 & .02 \\
\hline \multicolumn{13}{|c|}{ Target Present, Transfer } \\
\hline Set size 9 & 1,658 & 64 & .88 & .02 & .71 & .03 & 1,366 & 101 & .93 & .02 & .69 & .02 \\
\hline Set size 19 & 2,014 & 186 & .79 & .02 & .59 & .03 & 1,699 & 88 & .85 & .02 & .58 & .02 \\
\hline \multicolumn{13}{|c|}{ Target Absent, Transfer } \\
\hline Set size 9 & 3,908 & 320 & .99 & .01 & .74 & .02 & 2,955 & 230 & 1.00 & .00 & .75 & .02 \\
\hline Set size 19 & 4,637 & 433 & .98 & .00 & .61 & .02 & 3,461 & 240 & .98 & .01 & .60 & .01 \\
\hline \multicolumn{13}{|c|}{5 Exemplar } \\
\hline \multicolumn{13}{|c|}{ Target Present, Session 1} \\
\hline Set size 9 & 887 & 76 & .96 & .01 & .54 & .03 & 1,574 & 145 & .94 & .02 & .63 & .03 \\
\hline Set size 19 & 1,177 & 140 & .90 & .02 & .47 & .03 & 2,033 & 203 & .89 & .03 & .50 & .03 \\
\hline \multicolumn{13}{|c|}{ Target Absent, Session 1} \\
\hline Set size 9 & 1,394 & 190 & .99 & .00 & .54 & .04 & 4,615 & 744 & .99 & .00 & .68 & .03 \\
\hline Set size 19 & 2,397 & 406 & .98 & .01 & .49 & .03 & 5,104 & 864 & .99 & .01 & .54 & .03 \\
\hline \multicolumn{13}{|c|}{ Target Present, Session 2} \\
\hline Set size 9 & 669 & 31 & .95 & .01 & .63 & .03 & 1,300 & 132 & .93 & .01 & .69 & .03 \\
\hline Set size 19 & 898 & 90 & .94 & .01 & .49 & .03 & 1,594 & 189 & .89 & .02 & .58 & .03 \\
\hline \multicolumn{13}{|c|}{ Target Absent, Session 2} \\
\hline Set size 9 & 923 & 98 & .99 & .00 & .58 & .02 & 3,289 & 559 & .99 & .00 & .74 & .03 \\
\hline Set size 19 & 1,586 & 211 & .99 & .00 & .51 & .02 & 3,798 & 627 & 1.00 & .00 & .61 & .03 \\
\hline \multicolumn{13}{|c|}{ Target Present, Session 3} \\
\hline Set size 9 & 639 & 28 & .95 & .01 & .55 & .04 & 1,076 & 102 & .94 & .02 & .69 & .03 \\
\hline Set size 19 & 832 & 69 & .92 & .02 & .51 & .03 & 1,365 & 135 & .90 & .02 & .58 & .03 \\
\hline Target Absent, & & & & & & & & & & & & \\
\hline Set size 9 & 862 & 87 & .99 & .00 & .58 & .03 & 2,641 & 380 & .99 & .00 & .76 & .02 \\
\hline Set size 19 & 1,448 & 206 & .98 & .01 & .52 & .03 & 3,112 & 469 & .99 & .00 & .63 & .03 \\
\hline Target Present & & & & & & & & & & & & \\
\hline Set size 9 & 1,716 & 187 & .86 & .02 & .65 & .02 & 1,321 & 72 & .90 & .02 & .68 & .02 \\
\hline Set size 19 & 2,325 & 332 & .76 & .03 & .53 & .01 & 1,795 & 127 & .80 & .03 & .59 & .02 \\
\hline Target Absent, & & & & & & & & & & & & \\
\hline Set size 9 & 4,298 & 713 & .99 & .00 & .70 & .02 & 3,367 & 340 & .98 & .01 & .73 & .02 \\
\hline Set size 19 & 5,178 & 817 & .98 & .00 & .56 & .01 & 3,829 & 384 & .99 & .01 & .61 & .02 \\
\hline
\end{tabular}

Note-Data are broken down by target presence (present vs. absent) and set size ( 9 vs. 19).

tors and number of exemplars during training ( 5 vs. 20) and camouflage group (camouflage present or absent during training) as between-participants factors. Importantly, there was a main effect of camouflage training $[F(1,28)=$ $5.92, p<.05]$; participants trained on camouflage search displays found the target sooner when transferred to novel camouflage displays than did participants not trained on camouflage displays. Training conditions (camouflage vs. noncamouflage) also interacted with target presence $[F(1,28)=4.09, p=.05]$; camouflage training benefits were larger for target-absent trials. As for search slope, set size interacted with target presence and camouflage group $[F(1,28)=6.47, p<.05]$. The source of this interaction, according to Figure 4, appears to be a steeper search slope for the noncamouflage-trained group at transfer, especially when the target was absent (collapsed across ex- 


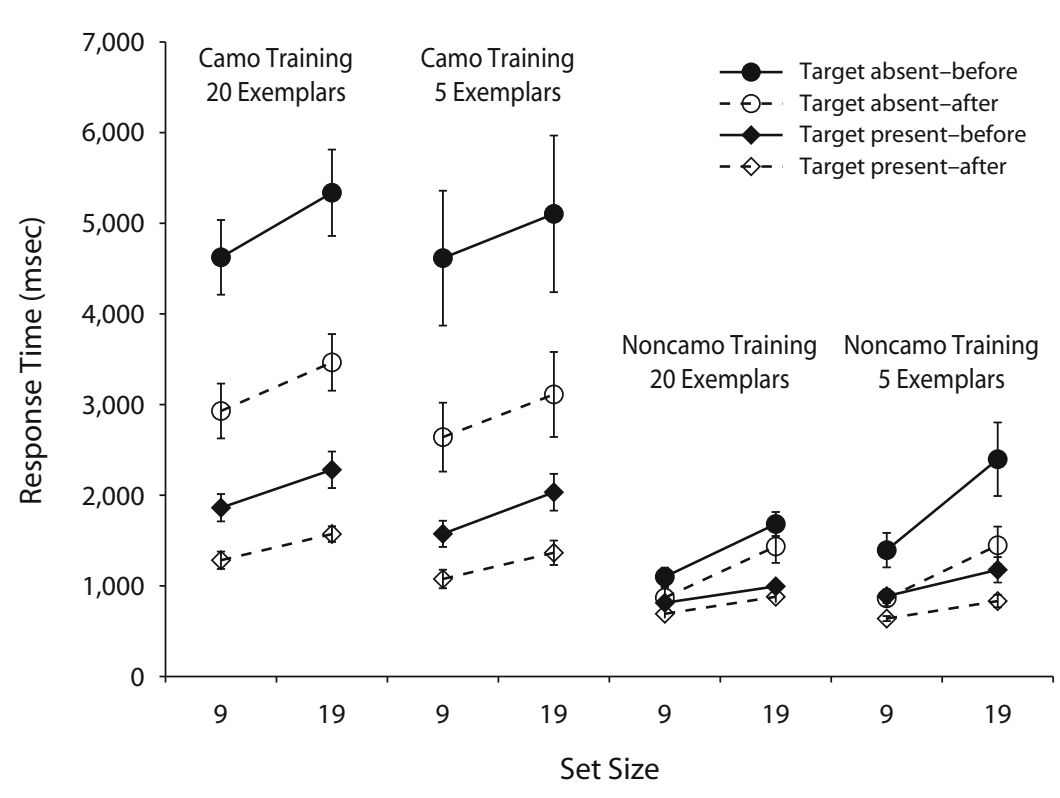

Figure 2. Pre- and posttraining response times for each group in Experiment 1. Error bars represent \pm 1 SEM. Camo, camouflage; noncamo, noncamouflage.

emplar number: camouflage-trained participants, 40 vs. $48 \mathrm{msec} /$ item for target present and absent, respectively; noncamouflage-trained participants, 48 vs. $80 \mathrm{msec} /$ item for target present and absent, respectively).

Accuracy data were entered into a similar ANOVA (Figure 5). At transfer, participants trained to search for camouflaged targets were more accurate when the target was present, as indicated by a significant camouflage $\times$ target presence interaction $[F(1,28)=4.58, p<.05]$. However, the number of exemplars participants trained on had no significant effect $[F(1,28)=7.61, p=.39]$ and did not interact significantly with any other factor (all $p \mathrm{~s}>.14$ ).

Was there a cost when participants were asked to search for new camouflaged targets? To answer this, we examined the large exemplar camouflage group that trained for three sessions on camouflaged targets and then were transferred to novel camouflaged targets in Session 4 (in this condition, the same number of targets were searched for during

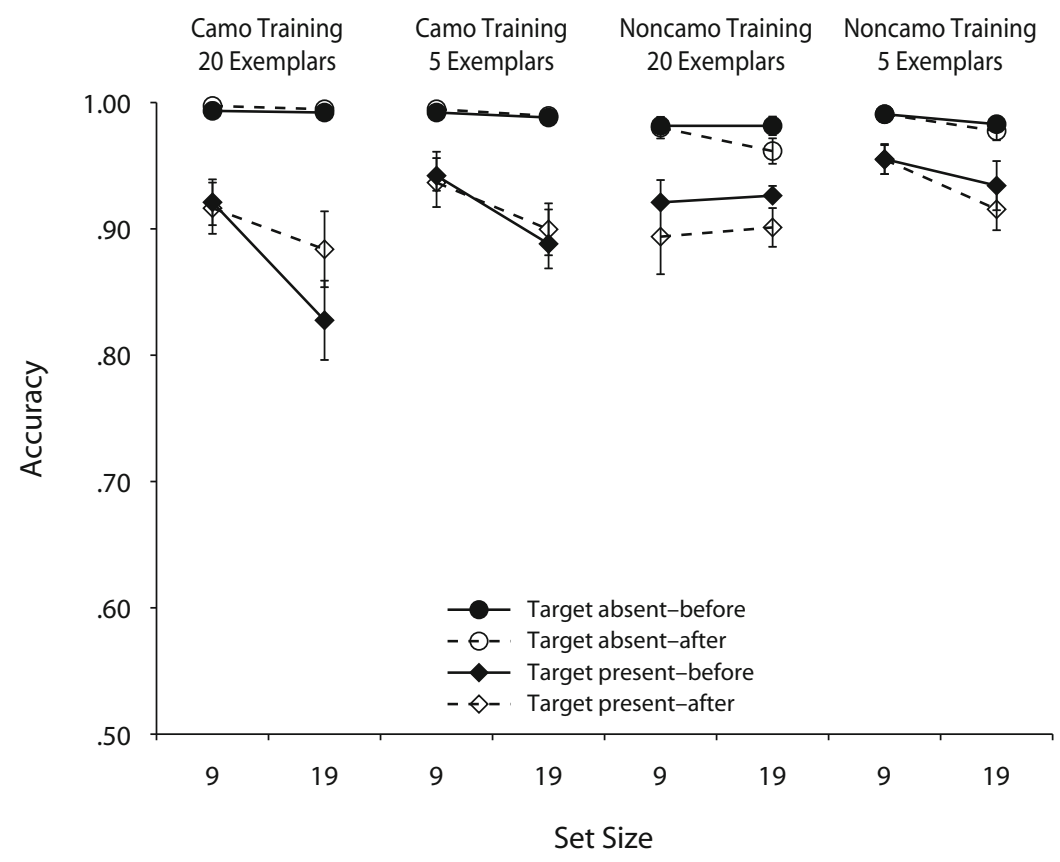

Figure 3. Pre- and posttraining accuracy for each group in Experiment 1. Error bars represent \pm 1 SEM. Camo, camouflage; noncamo, noncamouflage. 


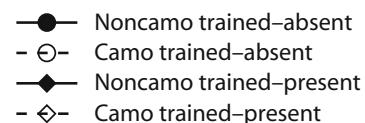

Transfer Session-Response Time

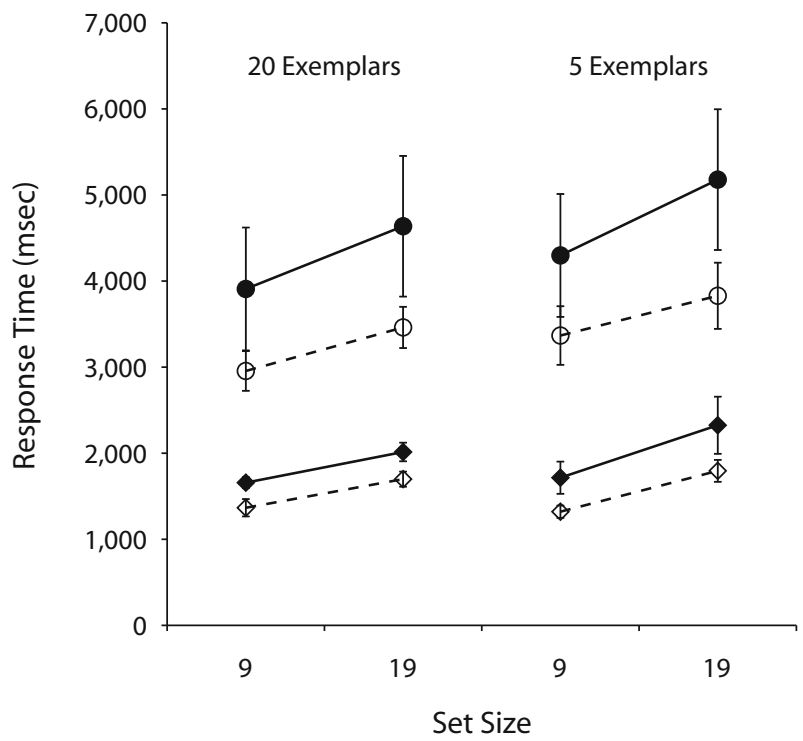

Figure 4. Response times at transfer for each group in Experiment 1. Error bars represent $\pm 1 S E M$. Noncamo, noncamouflage; camo, camouflage.

training and transfer, allowing a direct comparison to be made). We compared Session 3 (the last session of training) with Session 4, the transfer session. RT data were entered into an ANOVA, with session (3 vs. 4), set size, and target presence as factors. This ANOVA revealed no significant effect of session $[F(1,7)=0.14, p=.72]$, nor did session interact with target presence, set size, or target presence and set size (all $p \mathrm{~s}>.21$ ). Thus, the gains in RT were wholly preserved when camouflage-trained participants had to search for new camouflaged targets. Accuracy data were also entered into an identical ANOVA. Unlike for RTs, a small but nonstatistically significant effect was observed for session $[F(1,7)=4.06, p=.08]$, in addition to a trend for session and set size to interact $[F(1,7)=$ $4.25, p=.08]$. These trends were driven by a $2 \%$ drop in accuracy in the large set size condition. Although accuracy suffered slightly in the large set size condition at transfer, gains in RTs were preserved and could not be explained by a speed-accuracy trade-off (accuracy at transfer was greater than or equal to accuracy at Session 1).

\section{Fixations on Objects Versus Background}

We examined whether a shift from an "object-focused" strategy to a more "background-focused" strategy might be responsible for both improvement during practice and transfer (Figure 6). ${ }^{2}$ Focusing first on training (Session 1 vs. Session 3), an ANOVA was performed on the proportion of fixations that fell on discrete objects, with set size, session, and target presence as within-participants factors, and camouflage and exemplar number as the between-participants factor. This ANOVA revealed an interesting, but difficult to interpret, session $\times$ camouflage presence $\times$ exemplar number interaction $[F(1,28)=9.22$, $p<.01]$. Participants in the low exemplar number condition who were trained to search for camouflaged targets showed an increased focus on the background with training. However, a much smaller effect was observed in the high exemplar camouflage condition. Participants in the noncamouflage, high exemplar number condition actually showed a greater increase in background fixations than did the camouflage-trained high exemplar number group. A number of speculations can be made as to why this pattern of results was observed, but it is sufficiently interesting that large improvements can be observed without a major strategy shift. In the absence of a significant increase in background fixations, the high exemplar camouflage group demonstrated substantial and significant improvements in terms of both response speed and accuracy. Interestingly, even using strict criteria on whether a fixation fell on an object, participants in the camouflage groups fixated salient objects quite often (compared with the target-similar background); in some conditions, up to $40 \%-50 \%$ of the time. This $40 \%-50 \%$ does not capture many fixations that fell close to, but not on, objects or fixations directed between multiple objects (i.e., centerof-gravity fixations). As can be seen from Figure 6, even when no camouflage was present, participants fixated the background, likely as a result of these types of fixations. When 19 objects were in the search display, less than $20 \%$ of the search display was classified as containing an object; thus, it is especially surprising that so many eye movements were directed toward salient nontarget items.

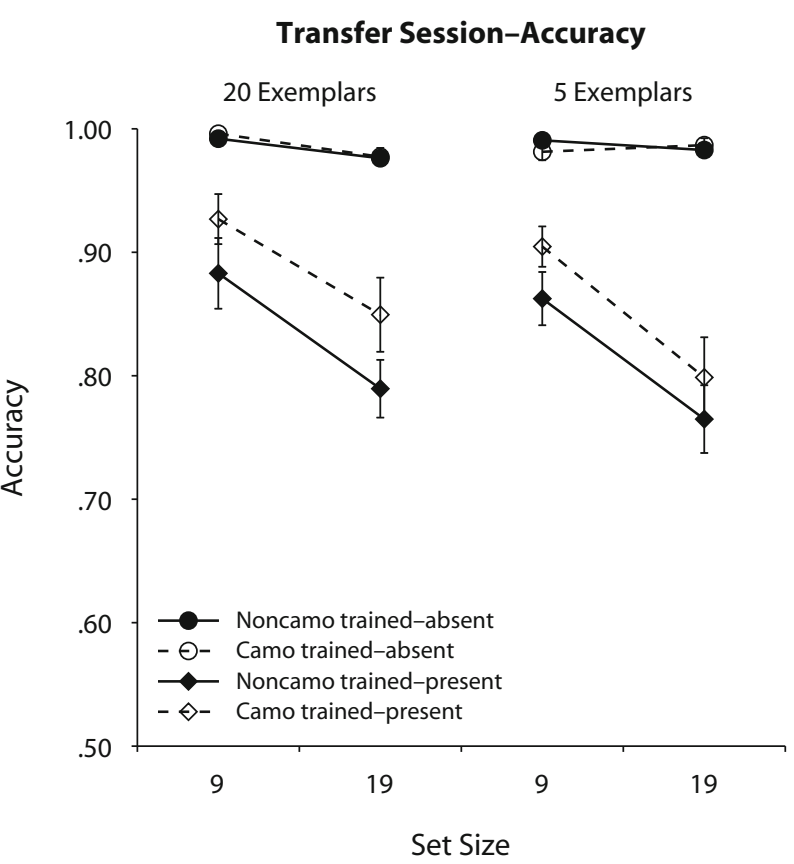

Figure 5. Accuracy at transfer for each group in Experiment 1. Error bars represent $\pm 1 S E M$. Noncamo, noncamouflage; camo, camouflage. 


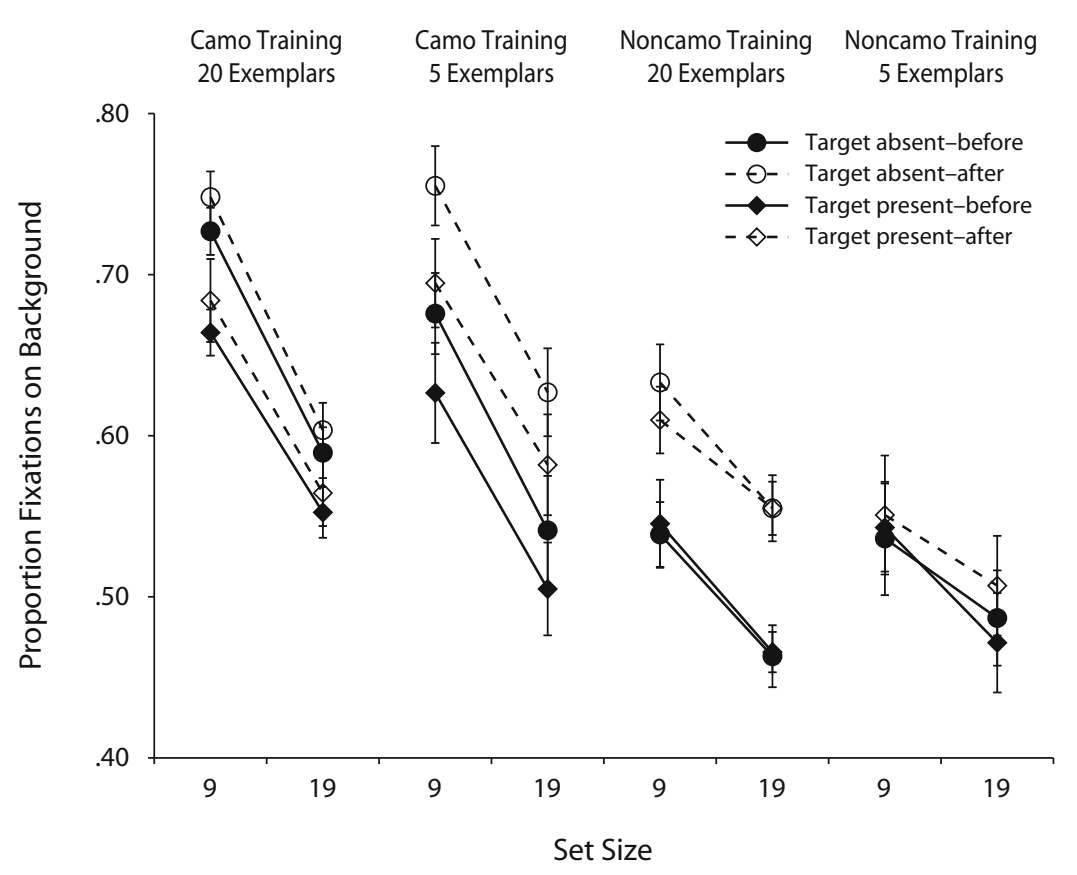

Figure 6. Proportion of fixations directed at the background for each group during training in Experiment 1. Error bars represent $\pm 1 S E M$. Camo, camouflage; noncamo, noncamouflage.

Similarly, when only 9 objects were in the display, less than $10 \%$ of the search display contained an object, yet participants fixated objects in many conditions between $20 \%$ and $35 \%$ of the time, depending on whether the target was absent or present.

Is an increased focus on the background the mechanism of transfer (Figure 7)? An ANOVA on the proportion of eye movements that fell on the background during Session 4 revealed no effect of camouflage group $[F(1,28)=$ $0.77, p=.21]$, and group did not interact with any other variable (all $p s>.23$ ). Thus, an increased focus on the background was not responsible for improved performance at transfer.

\section{Other Eye-Movement-Related Measures}

Six additional measures were investigated as the potential source of the benefit at transfer for those participants trained to find camouflaged targets. These measures included (1) average fixation duration, (2) the number of eye movements until the target was fixated (on trials on which the target was fixated), (3) RT after target fixation (on trials on which the target was fixated), (4) proportion of trials on which the target was fixated, (5) accuracy, if the target was fixated, and (6) accuracy, if the target was not fixated. Given that the mechanisms responsible for transfer are of primary interest, only eye movement measures that demonstrate significant transfer effects are depicted here; all other eye movement data can be found in an online appendix.

1. Average fixation duration was analyzed (see online appendix for data). From pre- to posttraining, there was a main effect of session $[F(1,28)=28.05, p<.001]$ and an interaction between session and camouflage group $[F(1,28)=10.84, p<.001]$. In general, fixation duration decreased with training, but did so more for participants searching camouflaged displays. Critically, at transfer, no significant difference was observed between participants who did and did not receive camouflage training $[F(1,28)=2.47, p=.13]$. The average difference in fixation duration at transfer between camouflage and noncamouflage groups was $15 \mathrm{msec}$. Thus, it does not appear that a decrease in fixation duration played a dominant role in performance differences between groups at the time of transfer.

2 . The number of eye movements required to first fixate the target (on trials on which the target was fixated) was also analyzed (Figure 8). From pre- to posttraining, there was a main effect of session $[F(1,28)=86.74, p<$ $.001]$ and an interaction between session and camouflage group $[F(1,28)=19.80, p<.001]$. Camouflage-trained participants showed a greater decrease in the number of eye movements required to fixate the target with training than did noncamouflage-trained participants. At transfer, camouflage-trained participants were able to fixate the target with fewer eye movements than noncamouflagetrained participants, even though targets, background, and distractors were novel $[F(1,28)=5.08, p<.05]$. The average difference in number of saccades at transfer between camouflage and noncamouflage groups was .9.

3 . The average time between a target being fixated and a buttonpress response being made (on trials on which the target was fixated and a correct response was made) was analyzed (Figure 9). From pre- to posttraining, there was a main effect of session $[F(1,28)=4.25, p<.001]$ 


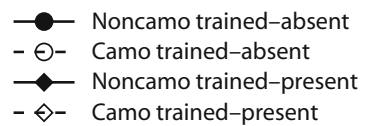

Transfer Session-Background Fixations

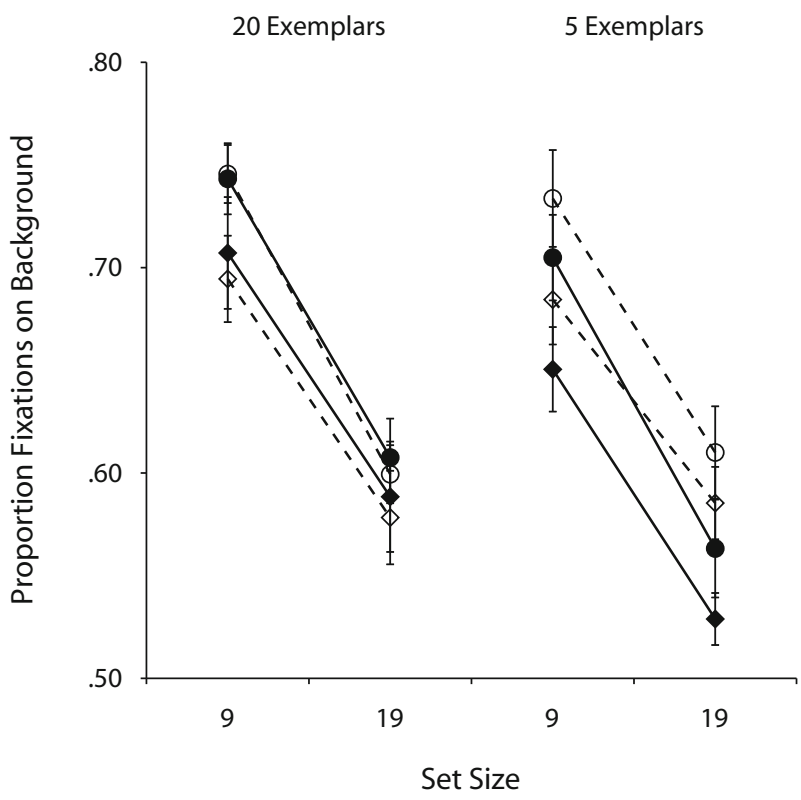

Figure 7. Proportion of fixations directed at the background at transfer for each group in Experiment 1. Error bars represent \pm 1 $S E M$. Noncamo, noncamouflage; camo, camouflage.

but no interaction between session and camouflage group session $[F(1,28)=0.35, p=.56]$. Thus, with training, all groups responded faster after the target was fixated. But this improvement only seemed evident for the camouflagetrained group at transfer. At transfer, camouflage-trained participants were significantly faster to respond, once their eyes landed on the target $[F(1,28)=11.03, p<$ $.01]$. The average difference in RT, given target fixation at transfer between camouflage and noncamouflage groups, was $120 \mathrm{msec}$.

4. The proportion of trials on which the target was fixated was analyzed (see online appendix). From preto posttraining, a complex interaction between set size, camouflage presence, and session emerged $[F(1,28)=$ $3.59, p=.07]$. Although this interaction did not reach statistical significance, it is interesting to note that the only group to show an increase in fixations on the target with training was the large exemplar (20) camouflage-trained group (when the set size was large). All other groups demonstrated a decrease in target fixations. At transfer, there were no significant main effects or interactions between training groups (all $p \mathrm{~s}>.26$ ). Thus, a change in the frequency of target fixation cannot explain differential performance differences at transfer.

5. Accuracy, if the target was fixated, remained relatively unchanged for all groups (see online appendix). From pre- to posttraining, there was no main effect of session $[F(1,28)=0.60, p=.60]$ and no interaction be- tween session and camouflage group session $[F(1,28)=$ $0.96, p=.34]$. At transfer, no significant differences existed between groups ( $p \mathrm{~s}>.16$ for all group effects and group interactions).

6. Accuracy, if the target was not fixated, was analyzed as well. From pre- to posttraining, there was a main effect of session $[F(1,28)=41.02, p<.001]$ and an interaction between session and camouflage group $[F(1,28)=5.60$, $p<.05]$. Camouflage-trained participants generally improved more (although within the small set size condition, training appeared to make little difference). However, at transfer, no significant differences existed between groups $(p>.10$ for all group effects and group interactions; see online appendix).

Saccades Until Target Fixation (Set Size: 9-Small)

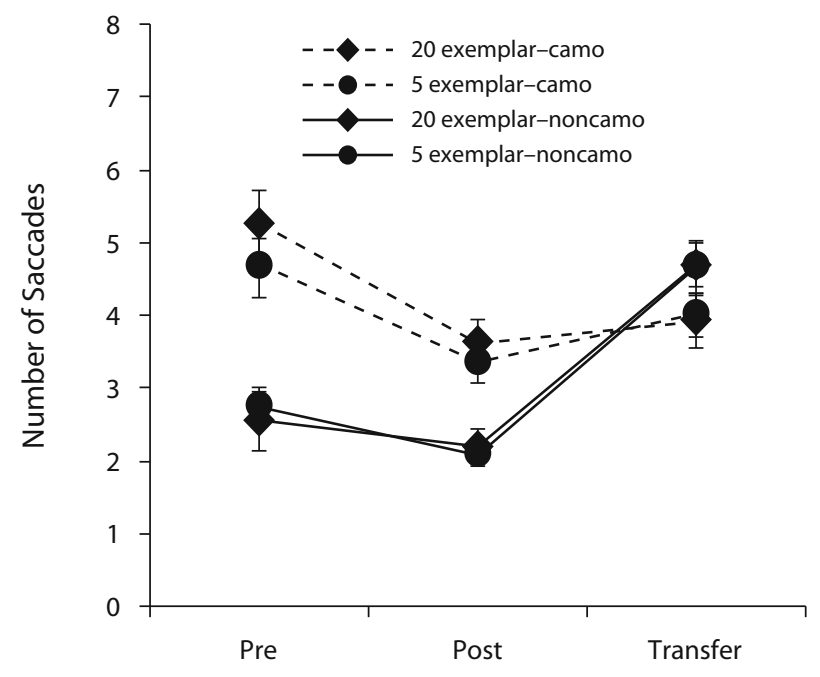

Saccades Until Target Fixation (Set Size: 19-Large)

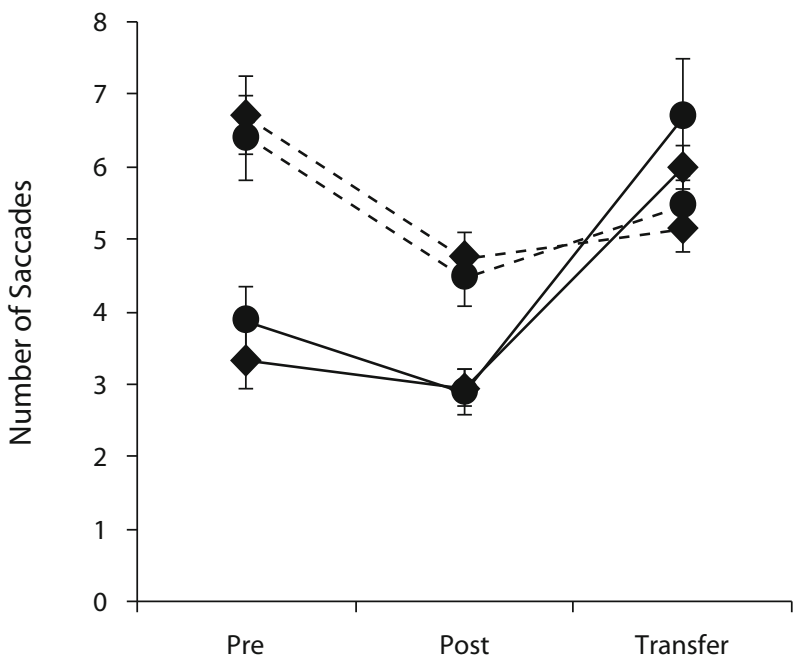

Figure 8. Number of saccades until the target is fixated before training, after training, and at transfer for each group in Experiment 1. Error bars represent $\pm 1 S E M$. Camo, camouflage; noncamo, noncamouflage. 
RT After Fixation (Set Size: 9-Small)

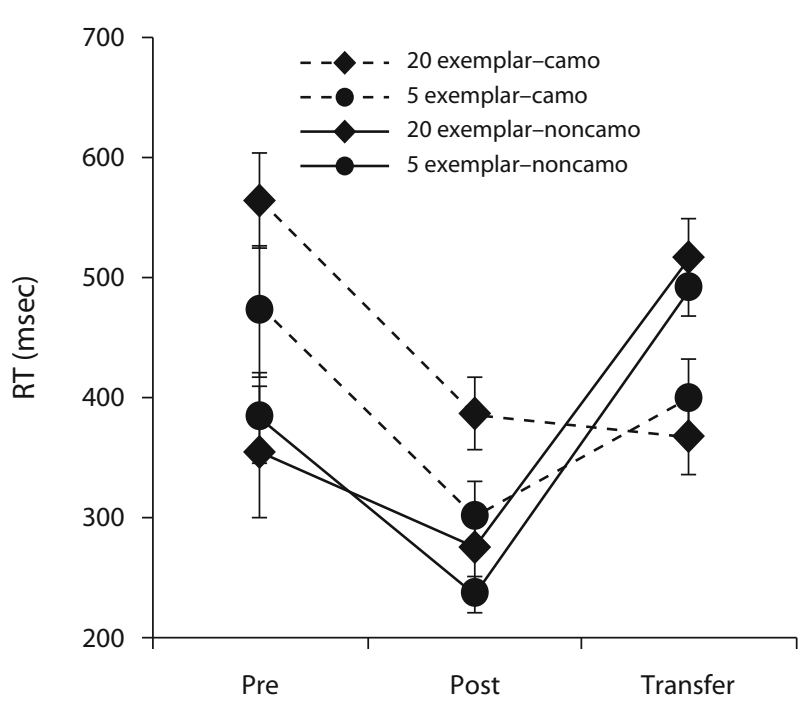

RT After Fixation (Set Size: 19-Large)

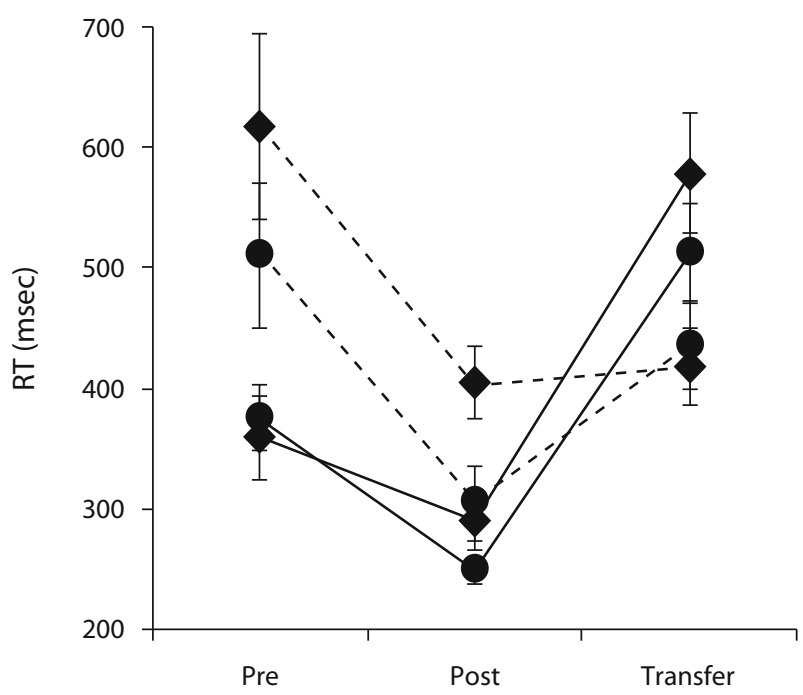

Figure 9. Time between fixation of the target and response for each group in Experiment 1. Error bars represent $\pm 1 S E M$. Camo, camouflage; noncamo, noncamouflage.

\section{Discussion}

In terms of RT and accuracy, participants trained to search for camouflaged targets outperformed participants trained to search for the same targets in noncamouflage situations when both groups were asked to search for novel camouflaged targets. At transfer, participants who had practiced searching for camouflaged targets were no more likely than those who had not to demonstrate greater overt attention to the background. Rather than an observable shift in scanning strategy, transfer seems largely related to measures that imply better recognition of target items, which allowed observers to get their eyes to the target with fewer eye movements and respond to it more quickly once they saw it. General target-background segmentation seems an unlikely explanation, given the lack of significant difference in overall saccade latencies at transfer (unless this improved segmentation is specific to segmenting targets from the background).

A direct comparison of camouflage-trained participants' performance during the last day of training and performance at transfer revealed no cost in terms of speed, and no significant cost in terms of accuracy; that is, participants seemed to have learned something general, rather than how specific targets stand out from specific backgrounds. Despite a nonsignificant trend to be less accurate at transfer than at the end of training, camouflagetrained participants showed significantly greater accuracy than noncamouflage-trained participants did at transfer; therefore, transfer of training to a completely novel set of search stimuli was nearly perfect.

After multiple hours of training, participants did not shift from a strategy of searching salient objects to a strategy of searching the background, even though the background was highly similar to the target in terms of features (see Figure 6, the camouflage-trained 20-exemplar group). Furthermore, participants trained to find camouflaged targets did not fixate the background at transfer any more than did participants who had not received camouflage training. But can performance be improved immediately by instructing participants to adopt a background search strategy (see Boot, Kramer, Becic, Wiegmann, \& Kubose, 2006, for a similar instructional manipulation)? If participants have a high degree of top-down control over their search, they should be able to direct their attention to less salient regions of the display to find the target. However, if search is largely driven by stimulus salience, participants should find this task difficult, and such manipulations might actually harm performance. These issues were examined in Experiment 2.

\section{EXPERIMENT 2}

In Experiment 2, participants were divided into three groups and completed one session of camouflage search to assess the degree to which the number of background fixations was amenable to instruction. One group was given no specific instructions about where to look for the target. One group was told that a common mistake participants make is to look at salient objects, and that they should search background regions instead. A third group, instructed to search the background, was given a diagram depicting the strategy we wanted them to use and completed a block of 20 practice trials before the experimental block of trials in which they were shown their own fixations superimposed on each search display.

\section{Method}

\section{Participants}

Twenty-four students at the University of Illinois participated in Experiment 2. All participants demonstrated normal or correctedto-normal acuity and color vision, and none participated in Experiment 1. Participants were paid $\$ 8$ for their participation in one 60 min session. 


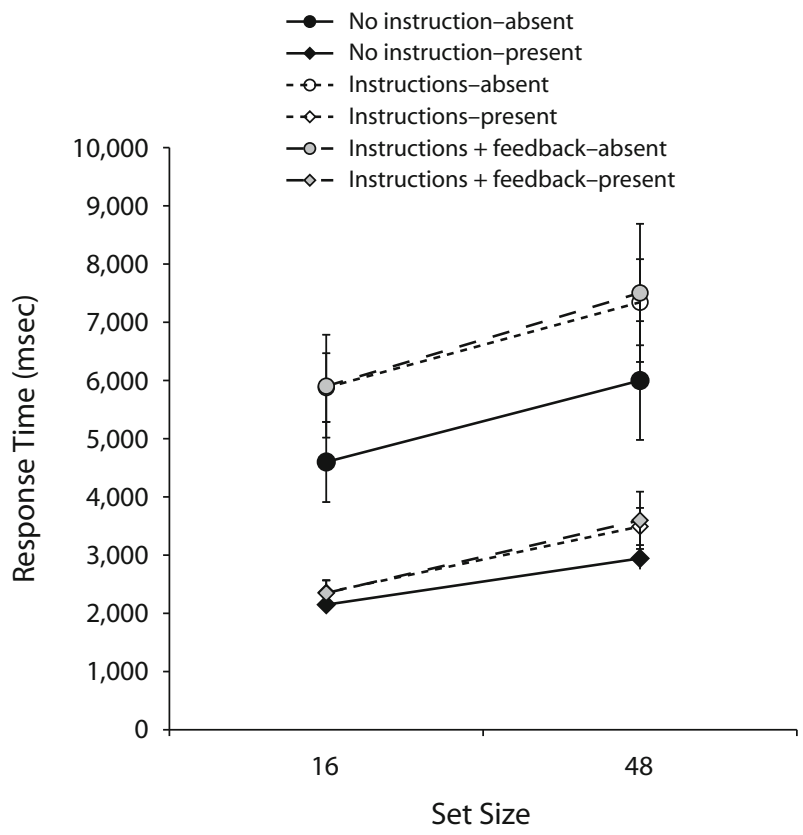

Figure 10. Response times for each group in Experiment 2. Error bars represent $\pm 1 S E M$.

\section{Apparatus and Stimuli}

The apparatus was the same as in Experiment 1. Stimuli were similar, except that larger set sizes were used (set sizes 16 and 48), and participants searched for 1 of 48 objects (instead of either 5 or 20 exemplars). Only camouflage search displays were used.

\section{Design and Procedure}

Participants completed one session of 400 trials. Participants were divided into the following three groups.

1. No-instruction group. Eight participants were given the same instructions as in Experiment 1. They were told to try to find the target quickly and accurately, but were not instructed on any particular strategy.

2. Instruction group. Eight participants were told that a common mistake participants make is to search the objects that stand out from the background. They were instructed that, since the target was always similar to the background, they should search the background rather than the objects that clearly stood out from it.

3. Instruction-plus-feedback group. Eight participants were given the same instructions that the instruction group had been given, but were also shown two diagrams of an example search display.

In each diagram were $\mathrm{Xs}$ that participants were told represented where a participant had searched (i.e., fixations). The first diagram depicted Xs on the objects in the search display. Participants were told that this was an inefficient strategy and were shown the search target actually hidden in the background, not where the participant had searched. The second diagram depicted the Xs away from the objects and on background regions (including the background region containing the target). Participants were told that this was the strategy we wanted them to use. Next, participants in this group completed 20 practice visual search trials. After each trial, participants were shown the search display again, with their fixations represented as black Xs on the display. Participants were told to attempt to maximize fixations on the background, as with the diagram they had been shown earlier. Afterward, all groups completed 400 trials of camouflage visual search.

It was predicted that search would be most efficient and accurate for participants instructed to use a background search strategy.

\section{Results}

The first 20 trials of the session were considered practice and were not included in the reported analyses, and neither were the 20 practice feedback trials the instructionplus-feedback group received. First, we focused on speed and accuracy of search, and next on the proportion of eye movements directed toward the background. Of critical interest was whether group (no instruction, instruction, instruction plus feedback) had an effect on these measures.

\section{RT and Accuracy}

RTs were entered into an ANOVA with set size (16 vs. 48) and target presence (absent vs. present) as withinparticipants factors and group (no instruction, instruction, instruction plus feedback) as a between-participants factor (Figure 10). Standard effects of set size $[F(1,21)=$ $79.92, p<.001]$, target presence $[F(1,21)=86.81, p<$ $.001]$, and the interaction between set size and target presence $[F(1,21)=10.01, p<.01]$ were observed. However, regardless of group, RTs were similar, as indicated by the lack of a significant group effect $[F(2,21)=0.85$, $p=.44]$. Group did not interact with set size, target presence, or set size and target presence (all $p \mathrm{~s}>.48$ ). Thus, instruction had no effect on RT or search efficiency. If anything, a focus on fixation location appeared to result in trends toward longer RTs and steeper slopes, possibly because of the additional load of attempting to implement a background-based strategy.

Accuracy data were entered into an identical ANOVA (Figure 11). There was an effect of target presence $[F(1,21)=175.18, p<.001]$ and set size $[F(1,21)=$

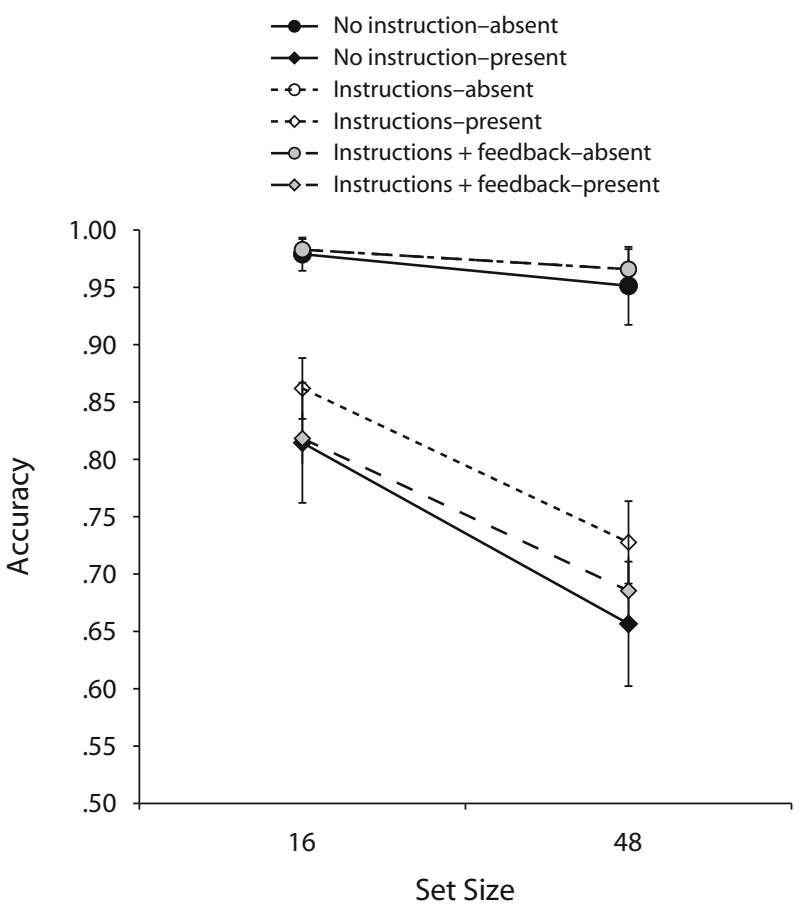

Figure 11. Accuracy for each group in Experiment 2. Error bars represent $\pm 1 S E M$. 


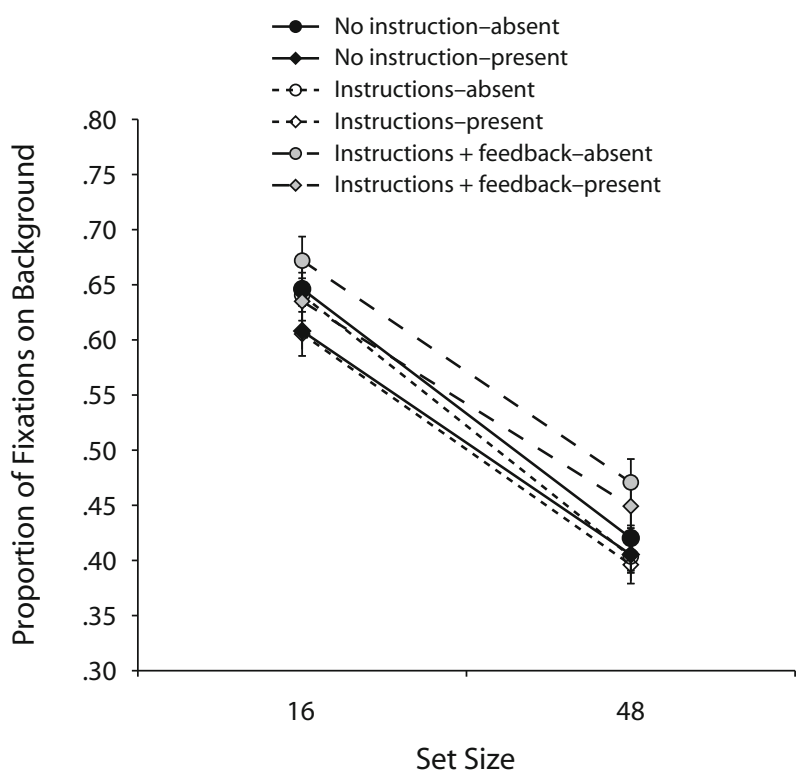

Figure 12. Proportion of fixations directed at the background for each group in Experiment 2. Error bars represent \pm 1 SEM.

107.91, $p<.001]$, and these two factors interacted $[F(1,21)=59.68, p<.001]$. However, as with RTs, there was no effect of group $[F(2,21)=0.47, p=.63]$, nor did group interact with any other factor or factors (all $p$ s $>$.40). Thus, instruction had no effect on response accuracy.

Finally, the proportion of eye movements on background regions was entered into an identical ANOVA (Figure 12). As expected, there was an effect of target presence $[F(1,21)=34.28, p<.001]$ and set size $[F(1,21)=$ $1136.39, p<.001]$, and an interaction between target presence and set size $[F(1,21)=6.89, p<.05]$. Importantly, there was no significant effect of group $[F(2,21)=$ $1.32, p=.29$ ], nor did group interact significantly with any other factor or factors (all $p s>.13$ ). Thus, in addition to having no effect on search speed or accuracy, explicit instructions on strategy had no effect on the proportion of fixations participants directed toward the target-similar background. More than half of all fixations were directed to salient items rather than to background regions in the large set size condition, regardless of instruction.

\section{Discussion}

Although stimulus factors such as set size and target presence had significant effects on the proportion of fixations directed to the background, explicit instruction (and feedback) to fixate background regions had no effect. Thus, participants seem to have been unable to exert much topdown control over their search and restrict it to background regions. As a result, performance, in terms of RTs, search slopes, and accuracy, did not differ. If participants were effective in allocating attention to the background, flat or even negative search slopes might have been expected (i.e., there is much more "background" to search in the small set size condition than in the large set size condition). However, this pattern was not observed. This is consistent with the findings of Experiment 1 that, at least in some camouflage conditions, multiple hours of training did not increase to a large extent the proportions of fixations on the background, and benefits at transfer seemed unrelated to a more backgroundfocused search strategy. Instead, other oculomotor measures appeared to be related to improved performance.

\section{GENERAL DISCUSSION}

How do observers become proficient at finding camouflaged targets? Our results suggest that, at least in terms of overt attention, this is not always accomplished by a strategy in which participants allocate more oculomotor attention to the background. In some of the camouflage search conditions, up to $50 \%$ or more of all fixations were devoted to searching salient objects rather than the background. In some cases, this object bias decreased slightly (e.g., the small exemplar camouflage group in Experiment 1), but there was no difference between camouflagetrained participants and noncamouflage-trained participants at transfer in terms of attention to the background.

Although our results suggest that a shift in attention allocation strategy is not responsible for improvements and transfer, it is important to recognize that our experiments can measure only overt attention. Covert attention (shifts of attention without an accompanying eye movement) may be allocated to the background to a greater degree with training, and this might account for some of the observed oculomotor and behavioral effects. However, given the tight coupling of covert and overt attention (e.g., Hoffman \& Subramaniam, 1995; Peterson, Kramer, \& Irwin, 2004), we believe this to be an unlikely explanation.

At transfer, camouflage-trained participants demonstrated a clear advantage, in terms of RTs and accuracy, over noncamouflage-trained participants. This is somewhat surprising, given that much of the perceptual learning literature would suggest that transfer of training should be fairly limited (e.g., Ahissar \& Hochstein, 1996; Ball \& Sekuler, 1982; Ellison \& Walsh, 1998; Fiorentini \& Berardi, 1981). Yet in our experiments, transfer was found to be nearly perfect. Eye-movement analyses suggest two reasons for benefits at transfer: (1) Camouflage-trained participants are able to fixate novel camouflaged targets with fewer eye movements; and (2) such participants are able to respond faster when their eyes land on the target. These findings suggest that camouflage-trained observers are learning something general about how to find camouflaged targets, not how certain targets stand out against certain backgrounds. Specifically, we propose that participants are learning something general about target recognition, which allows them to guide their eyes to the target more efficiently and recognize it faster and farther from fixation. For example, participants could have learned that slight discontinuities in the background are worthy of further inspection, even though this did not lead to an overall strategy of devoting more oculomotor fixations to background regions that did not contain such discontinuities. On a related note, participants could have learned to take advantage of the regularity of the background created 
by tiling pieces of the target, and learned that deviations in this regularity signified the presence of a target.

The present results are largely consistent with the results of McCarley, Kramer, Wickens, Vidoni, and Boot (2004). McCarley and colleagues studied training and transfer of training in the context of another difficult visual search task: screening of X-ray images of luggage. With extensive practice, observers fixated the target with fewer eye movements and recognized it more quickly when it was fixated. However, transfer in the present article would appear to be even broader than the transfer observed in the luggage screening task. In our transfer tasks, the shapes and colors of targets were often different from those of the training stimuli, whereas the knives used in the luggage screening search task were arguably more homogeneous.

The data presented here have implications for both theories and models of attention and visual search. To a large extent, these data are consistent with salience-driven models of visual attention (e.g., Carmi \& Itti, 2006; Itti \& Koch, 2000). A substantial proportion of eye movements were directed toward salient objects rather than toward background regions similar to the target. In Experiment 2, instructions to search the background rather than the objects that stood out from the background had no effect. These results are also broadly consistent with the findings of Sobel and Cave (2002), in which an instructional manipulation did not result in participants restricting their search for a conjunction target to a specific relevant feature. At first glance, it would appear to be difficult, using search models based on top-down guidance of attention, to explain the object bias observed here. The influential Guided Search 2.0 model posits separate feature maps that feed into a master activation map, with activation in the master map determining the order in which items are searched (Wolfe, 1994). Top-down knowledge of the features that define the target increases activation within the relevant feature maps (and thus the master map) to allow attention to be guided to the target. But how would such a model operate when the visual features defining the target overlapped with the background? Would search for a red car on a red background among toys of many other colors result in many background fixations? Perhaps not. A sophisticated searcher might recognize the noninformative nature of this feature, and instead guide attention on the basis of a more informative visual property. For example, the searcher might recognize that the target is always a discontinuity or break in the camouflage background and use this information to guide attention. Although this cue is informative, the most salient instances of discontinuities in the background are in fact the distractor objects. Thus, a number of nontarget objects would be fixated before the target is discovered, as was the case in the data presented here.

The target acquisition model (TAM) would appear to have similar difficulties predicting search behavior under conditions of camouflage (Zelinsky, 2008). TAM is a fixed-parameter computational model of eye movements that can successfully predict human fixations in a range of both simple and complex visual search situations (from a search for an $\mathrm{O}$ among Qs to a search for a tank in a realistic landscape scene). TAM predicts fixation locations, and their order, on the basis of visual similarity between regions of the search display (transformed to take acuity limitations into account) and the search target. However, under conditions of camouflage, much of the background is extremely similar to the search target, which would presumably make efficient guidance on the basis of visual similarity difficult for TAM. Why is it then that participants tended to fixate salient distractor objects rather than the background that was highly correlated with the target? As Zelinsky says (2008, p. 827), like most image-based models of search, all regions of a scene (objects and background regions) are treated equally by TAM; that is, TAM does not take into consideration the special status of objects in visual processing. Zelinsky suggests the addition of an initial object-segmentation step to improve the utility of TAM and other models in predicting attention and eye movements during camouflage search.

Our results are consistent with conceptions of visual attention in which objects are the unit on which attention acts (e.g., Duncan, 1984; Kramer \& Watson, 1996; Vecera \& Farah, 1994). Specifically regarding attention and search, the present data are consistent with data showing that objects are the basic unit of analysis during visual search (Wolfe \& Bennett, 1997). It should be noted that the object bias in the present set of data is actually an underestimate of participants' tendency to fixate objects. A fixation was only counted as being directed toward an object if it fell on an object. In many cases, the eyes were directed toward an object but did not fall directly on it. Other fixations appeared to be "center-of-mass" fixations directed toward the middle of groups of objects. This pattern of on-object versus off-object fixations was largely consistent, even after many hundreds of trials of training, and even after explicit instruction to avoid on-object fixations. Thus, Neider and Zelinsky's (2006a) suggestion that camouflaged targets should "hide in plain sight" to avoid detection is valid after extensive training.

A number of questions remain unanswered and should be the topic of future research. Interestingly, the fact that some participants in Experiment 1 trained on four times as many exemplars as did others did not improve performance at transfer; or if it did, such improvement was not specific to the camouflage condition. It is possible that only five targets were needed to engender broad transfer to novel targets, but it would be interesting to see whether a greater difference between the numbers of exemplars trained on would affect the degree of transfer. Also, our attempts to manipulate strategy were unsuccessful. It is possible that other forms of feedback (e.g., continuous trial-by-trial feedback on the proportion of on- vs. offobject fixations) might be more effective. However, the present results suggest an object bias that may be difficult or impossible to overcome.

\section{AUTHOR NOTE}

The authors thank Jeremy Wolfe, Kyle Cave, and an anonymous reviewer for their helpful comments and suggestions on an earlier version of 
this manuscript. We also thank the Office of Naval Research for their support of this research (N00014-07-1-0903) in the form of a MURI award. Correspondence concerning this article should be addressed to W. R. Boot, Department of Psychology, Florida State University, 1107 W. Call Street, Tallahassee, FL 32306-4031 (e-mail: boot@psy.fsu.edu).

\section{REFERENCES}

Ahissar, M., \& Hochstein, S. (1996). Learning pop-out detection: Specificities to stimulus characteristics. Vision Research, 36, 34873500.

BACON, W. F., \& EgEth, H. E. (1994). Overriding stimulus-driven attentional capture. Perception \& Psychophysics, 55, 485-496.

Ball, K., \& SEKUler, R. (1982). A specific and enduring improvement in visual motion discrimination. Science, 218, 697-698.

Boot, W. R., Kramer, A. F., Becic, E., Wiegmann, D. A., \& Kubose, T. (2006). Detecting transient changes in dynamic displays: The more you look, the less you see. Human Factors, 48, 759-773.

Boot, W. R., McCarley, J. S., Kramer, A. F., \& Peterson, M. S. (2004). Automatic and intentional memory processes in visual search. Psychonomic Bulletin \& Review, 11, 854-861.

Brockmole, J. R., \& Henderson, J. M. (2006). Using real-world scenes as contextual cues for search. Visual Cognition, 13, 99-108.

CARMI, R., \& ITTI, L. (2006). Visual causes versus correlates of attentional selection in dynamic scenes. Vision Research, 46, 4333-4345.

Chen, X., \& Zelinsky, G. J. (2006). Real-world visual search is dominated by top-down guidance. Vision Research, 46, 4118-4133.

ChUn, M., \& JiANG, Y. (1998). Contextual cuing: Implicit learning and memory of visual context guides spatial attention. Cognitive Psychology, 36, 28-71.

Clarke, A. D. F., Green, P. R., Chantler, M. J., \& Emrith, K. (2008). Visual search for a target against a $1 / f^{\beta}$ continuous textured background. Vision Research, 48, 2193-2203.

Duncan, J. (1984). Selective attention and the organization of visual information. Journal of Experimental Psychology: General, 113, 501-517.

Ellison, A., \& WALSH, V. (1998). Perceptual learning in visual search: Some evidence of specificities. Vision Research, 38, 333-345.

FioRentini, A., \& BERARDI, N. (1981). Learning in grating waveform discrimination: Specificity for orientation and spatial frequency. $V i-$ sion Research, 21, 1149-1158.

Folk, C. L., Remington, R. W., \& Johnston, J. C. (1992). Involuntary covert orienting is contingent on attentional control settings. Journal of Experimental Psychology: Human Perception \& Performance, 18, 1030-1044.

Hoffman, J. E., \& Subramaniam, B. (1995). The role of visual attention in saccadic eye movements. Perception \& Psychophysics, 57, 787-795.

IтTI, L., \& KoсH, C. (2000). A saliency-based search mechanism for overt and covert shifts of visual attention. Vision Research, 40, 1489-1506.

KLeIN, R. M., \& MacInNes, W. J. (1999). Inhibition of return is a foraging facilitator in visual search. Psychological Science, 10, 346-352.

Kramer, A. F., \& Watson, S. E. (1996). Object-based visual selection and the principle of uniform connectedness. In A. F. Kramer, M. G. H. Coles, \& G. Logan (Eds.), Converging operations in the study of visual attention (pp. 395-414). Washington, DC: American Psychological Association.

McCarley, J. S., Kramer, A. F., Wickens, C. D., Vidoni, E. D., \& Воот, W. R. (2004). Visual skills in airport security screening. Psychological Science, 15, 302-306.

MotTer, B., \& BelKy, E. (1998). The guidance of eye movements during active visual search. Vision Research, 38, 1805-1815.
Neider, M. B., \& Zelinsky, G. J. (2006a). Scene context guides eye movements during search. Vision Research, 46, 614-621.

NeIDER, M. B., \& ZelinSKY, G. J. (2006b). Searching for camouflaged targets: Effects of target-background similarity on visual search. $\mathrm{Vi}$ sion Research, 46, 2217-2235.

Peterson, M. S., Kramer, A. F., \& Irwin, D. E. (2004). Covert shifts of attention precede involuntary eye movements. Perception \& Psychophysics, 66, 398-405.

Proulx, M. (2007). Bottom-up guidance in visual search for conjunctions. Journal of Experimental Psychology: Human Perception \& Performance, 33, 48-56.

Sobel, K. V., \& CAVE, K. R. (2002). Roles of salience and strategy in conjunction search. Journal of Experimental Psychology: Human Perception \& Performance, 28, 1055-1070.

Theeuwes, J. (1992). Perceptual selectivity for color and form. Perception \& Psychophysics, 51, 599-606.

TheEuwes, J. (2004). Top-down search strategies cannot override attentional capture. Psychonomic Bulletin \& Review, 11, 65-70.

Vecera, S. P., \& Farah, M. J. (1994). Does visual attention select objects or location? Journal of Experimental Psychology: General, 123, 146-160.

WoLfe, J. M. (1994). Guided Search 2.0: A revised model of visual search. Psychonomic Bulletin \& Review, 1, 202-238.

Wolfe, J. M., \& Bennett, S. C. (1997). Preattentive object files: Shapeless bundles of basic features. Vision Research, 37, 25-44.

Wolfe, J. M., Oliva, A., Horowitz, T. S., Butcher, S. J., \& BomPAS, A. (2002). Segmentation of objects from backgrounds in visual search tasks. Vision Research, 42, 2985-3004.

Yantis, S., \& Jonides, J. (1984). Abrupt visual onsets and selective attention: Evidence from visual search. Journal of Experimental Psychology: Human Perception \& Performance, 10, 601-621.

ZeLINSKY, G. J. (1996). Using eye saccades to assess the selectivity of search movements. Vision Research, 36, 2177-2187.

ZELINSKY, G. J. (2008). A theory of eye movements during target acquisition. Psychological Review, 115, 787-835.

\section{NOTES}

1. Four five-item training sets were created from Set A and Set B, for a total of eight separate five-item training sets. One five-item set was assigned to each of 8 participants in the 5-exemplar camouflage group and the 5-exemplar noncamouflage group. Thus, target properties were equated across the 5-examplar camouflage and noncamouflage groups, and across participants, those in the 5-exemplar groups had experience with all of the same items participants in the 20-exemplar group had.

2. A stricter criterion was used to determine whether or not the eyes fell on an object, compared with the criteria used by Neider and Zelinsky (2006a), resulting in fewer eye movements being classified as on-object ( 40 vs. 50 pixels). By classifying more of the display as "background," we maximize our ability to detect subtle shifts in strategy from an objectsearch strategy to a background-search strategy.

\section{SUPPLEMENTAL MATERIALS}

An appendix containing supplemental eye movement data, in Adobe .pdf format, can be downloaded from app.psychonomic-journals.org/ content/supplemental.

(Manuscript received June 8, 2008; revision accepted for publication December 29, 2008.) 